\title{
Caracterización clínico epidemiológica de las cardiopatías congénitas en niños. Instituto Hondureño de Seguridad Social.
}

\author{
Clinical epidemiological characterization of congenital heart disease in childrenat the \\ Honduran Social Security Institute
}

Paola Sophia Bonilla Medina, * Christian Leonel Breuillet Barrera,* Juan Rafael Guerrero Manueles. **

\section{RESUMEN}

Antecedentes: Las malformaciones congénitas que más frecuentemente determinan malformaciones infantiles son las cardiopatías, producen $30 \%$ de las muertes en pacientes pediátricos. Desde que en 1939 Robert Gross realizó la primera intervención quirúrgica de un paciente con cardiopatía congénita (CC), éstas han cobrado gran importancia para la medicina y la salud pública. Objetivo: del estudio fue caracterizar clínica y epidemiológicamente los pacientes que presentaron cardiopatías congénitas en la consulta externa de cardiología del Hospital Regional del NorteInstituto Hondureño de Seguridad Social (HRNIHSS) durante el período de enero a octubre del 2015. Pacientes y Métodos: Se realizó un estudio de enfoque cuantitativo, descriptivo transversal en la consulta externa HRN-IHSS, la población fue de 859 pacientes atendidos y la muestra 551 pacientes en quienes se confirmó la presencia de cardiopatía. Los datos se obtuvieron de los informes de atenciones diarias entre los meses de enero a octubre del 2015. Resultados: Se obtuvieron 551 pacientes con cardiopatías congénitas. 294 (53.35\%) de los pacientes eran del género masculino y 257 (46.65\%) del género femenino. 265(48.1\%) estaban en edades comprendidas entre 1-2 años. 191 (34.66\%) de los niños tenían comunicación interauricular (CIA). Conclusiones: $\mathrm{EI}$ género masculino y los lactantes fueron los más afectados por cardiopatías congénitas.

\footnotetext{
*Residente de segundo año de Pediatría. Escuela Universitaria de Ciencias de la Salud, Universidad Nacional Autónoma de Honduras en el Valle de Sula EUCS/UNAH-VS

**Cardiólogo Pediatra Instituto Hondureño de Seguridad Social IHSS Dirigir correspondencia a: paolasophia@gmail.com, cbreuillet@hotmail.com, rafaelguerrero75@yahoo.es

Recibido: 17 de noviembre 2015 Aprobado: 15 de marzo 2016
}

\section{PALABRAS CLAVE}

Anomalías congénitas, Cardiopatía, Niños.

\section{ABSTRACT}

Background: Congenital malformations that most frequently determine infant malformations are heart malformations, which cause $30 \%$ of deaths in pediatric patients. Robert Gross performed the first surgery in a patient with congenital heart disease (CHD) in 1939. Since then, it has become a very important procedure in medicine and public health. The objective of the study was to characterize clinically and epidemiologically patients who had CHD at the Northern Regional Hospital of the Honduran Social Security Institute (IHSS-HRN) from January to October 2015. Patients and Methods: a descriptive study was conducted at the HRN- IHSS, the universe was 859 patients seen and the sample was 551 patients in whom the presence of CHD was confirmed. The data was obtained from daily reports attentions between the months of January to October 2015. Results: There were 551 patients with CHD. 294 (53.35\%) patients were male and 257 (46.65\%) were female. 265 (48.1\%) had 1-2 years of age. 191 (34.66\%) of the children had atrial septal defect (ASD). Conclusions: CHD affects males and the diagnosis is made in the first two years of life.

\section{KEYWORDS}

Children, Congenital anomalies, Heart disease.

\section{INTRODUCCIÓN}

Cardiopatía congénita se define como una anormalidad en la estructura y/o función del corazón en el recién nacido, establecida durante la gestación. En general, las cardiopatías congé- 
nitas corresponden a malformaciones del corazón resultantes de un desarrollo embrionario alterado. $^{(1-3)}$ Las malformaciones congénitas abarcan una amplia variedad de alteraciones del desarrollo fetal. La herencia multifactorial es responsable de la mayoría de las malformaciones mayores. Afectan al 2-3\% de los recién nacidos al momento del parto, aunque al final del primer año de vida se detectan hasta en un 7\%. ${ }^{(4,5)} \mathrm{La}$ incidencia mundial de defectos congénitos oscila entre 25 y $62 / 1,000$ al nacimiento, y al menos 53/1,000 individuos tienen una enfermedad con vínculo genético que se manifiesta antes de los 25 años. ${ }^{(5,6)}$

Las malformaciones congénitas que más frecuentemente determinan malformaciones infantiles son las cardiopatías congénitas, que producen $30 \%$ de las muertes. ${ }^{(7)}$ Desde que, en 1939, Robert Gross realizó la primera intervención quirúrgica de un paciente con cardiopatía congénita (CC), éstas han cobrado gran importancia para la medicina y la salud pública. ${ }^{(8)}$

En este sentido, resulta necesario conocer las características epidemiológicas de las cardiopatías congénitas, a fin de determinar cuántos cardiólogos pediatras, servicios asistenciales y demás recursos son necesarios para afrontar adecuadamente la atención que requieren los pacientes con estas afecciones. No existen en la Institución estudios de base poblacional o epidemiológica que permitan una aproximación al conocimiento de la magnitud y distribución por tipo de cardiopatías congénitas. Más allá de la utilidad de este trabajo, el propósito es alertar acerca de la importancia de las cardiopatías congénitas en nuestro país, así como impulsar al desarrollo de investigaciones que permitan un conocimiento más exacto y directo de nuestra realidad en este campo.

\section{PACIENTES Y MÉTODOS}

Se realizó un estudio de enfoque cuantitativo, en la consulta externa de cardiología pediátrica del Instituto Hondureño del Seguro Social, de la ciudad de San Pedro Sula, Cortés, Honduras, durante el período de enero a octubre del 2015.
Estudio con diseño no experimental y alcance descriptivo. La población estuvo constituida por 859 pacientes atendidos en la consulta externa de cardiología pediátrica, en el período descrito. El muestreo fue por conveniencia, incluyéndose a 551 pacientes en quienes se confirmó la presencia de cardiopatía. Los datos incluidos de la caracterización fueron obtenidos de las atenciones diarias, comprendían; total de pacientes, tipos de cardiopatías, edad, género, casos nuevos y subsiguientes. Los criterios de inclusión comprendieron todos los pacientes con cardiopatías; los criterios de exclusión comprendían los pacientes que se presentaron por sospecha de cardiopatía pero resultaron sin alteración. El análisis estadístico se realizó aplicando las medidas de frecuencia, tasas y proporciones. Se utilizó el programa Microsoft Excel 2010.

\section{RESULTADOS}

Se encontró que de los 551 pacientes con cardiopatías congénitas, 294 (53.35\%) eran del género masculino y 257 (46.65\%) del género femenino. En cuanto a la edad, se encontró que $265(48.1 \%)$ pacientes tenían entre 1 mes y 2 años, 126 (22.86\%) tenían entre 3- 5 años. (Ver Gráfico No. 1)

\section{Gráfica No. 1: Distribución de los pacientes según edad.}

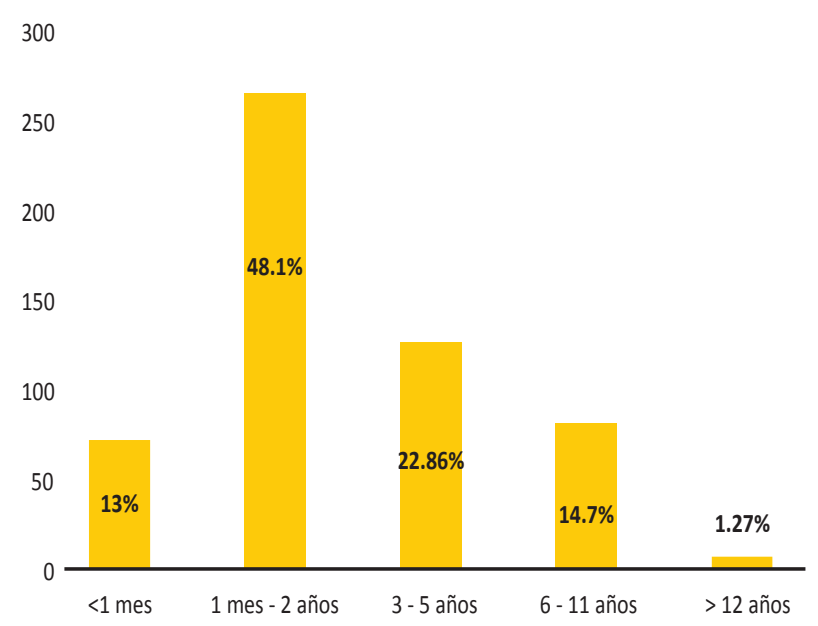

Fuente: Investigación cardiopatías congénitas. 
En cuanto a los tipos de cardiopatía encontradas; comunicación interauricular (CIA) 191 (34.66\%) pacientes, comunicación interventricular (CIV) 125 (22.7\%), persistencia del conducto arterioso (PCA) 117 (21.23\%) pacientes, y tetralogía de Fallot 39 (7.07\%) pacientes. (Ver tabla No. 1.)

Tabla No. 1: Distribución de los pacientes según tipo de cardiopatía.

\begin{tabular}{|c|c|c|}
\hline Cardiopatía & $\mathbf{n}$ & Porcentaje \\
\hline$-\mathrm{CIA}$ & 191 & 34.66 \\
\hline$* \mathrm{CIV}$ & 125 & 22.7 \\
\hline$+\mathrm{PCA}$ & 117 & 21.23 \\
\hline TETRALOGIA DE FALLOT & 39 & 7.07 \\
\hline COARTACION DE LA AORTA & 32 & 5.8 \\
\hline DOBLE TRACTO DE SALIDA DEL & 9 & 1.63 \\
\hline \multicolumn{3}{|l|}{ VENTRICULO DERECHO } \\
\hline CANAL AURICULO VENTRICULAR & 8 & 1.45 \\
\hline \multicolumn{3}{|l|}{ COMPLETO } \\
\hline ESTENOSIS DE VALVULA AORTICA & 6 & 1.1 \\
\hline ATRESIA TRICUSPIDEA & 6 & 1.1 \\
\hline ESTENOSIS VALVULA PULMUNAR & 5 & 0.9 \\
\hline DEXTROCARDIA & 4 & 0.72 \\
\hline VENTRICULO UNICO & 2 & 0.36 \\
\hline HIPERTENSION ARTERIAL & 2 & 0.36 \\
\hline \multicolumn{3}{|l|}{ PULMONAR } \\
\hline FORAMEN OVAL PERMEABLE & 2 & 0.36 \\
\hline MEMBRANA SUBVALVULAR & 2 & 0.36 \\
\hline \multicolumn{3}{|l|}{ AORTICA } \\
\hline SITUS INVERSO & 1 & 0.2 \\
\hline TOTAL & 551 & $100 \%$ \\
\hline
\end{tabular}

*CIV: Comunicación interventricular, + PCA: persistencia del conducto arterioso, - CIA: comunicación interauricular Fuente: Investigación cardiopatías congénitas

En relación al número de consultas, se encontró que 137 (24.80\%) de los pacientes eran atendidos por primera vez y 414(75.2\%) eran subsiguientes. Se determinó la distribución de la cardiopatías en los 27 pacientes con Síndrome de Down, encontrando que 11 (40.7\%) tenían comunicación auricular junto con persistencia del conducto arterioso (Ver tabla No. 2). Se realizó intervención quirúrgica en 35(6.35\%) de los 551 pacientes. (Ver tabla No. 3)
Tabla No. 2: Distribución de las cardiopatías en pacientes con Síndrome de Down.

\begin{tabular}{lcc}
\hline TIPO DE & $\mathrm{n}$ & Porcentaje \\
CARDIOPATIA & 7 & $26 \%$ \\
\hline${ }^{*}$ CIV & 1 & $3.7 \%$ \\
+PCA & 4 & $14.8 \%$ \\
-CIA & 11 & $40.7 \%$ \\
CIA+ PCA & 1 & $3.7 \%$ \\
CIV+ TETRALOGIA DE FALLOT & 2 & $7.4 \%$ \\
CANAL AURICULOVENTRICULAR COMPLETO & 1 & $3.7 \%$ \\
HIPERTENSION ARTERIAL PULMONAR & 27 & $100 \%$ \\
Total & 27 \\
*CIV: Comunicación interventricular, + PCA: persistencia del \\
Conducto arterioso, - CIA: comunicación interauricular \\
Fuente: Investigación cardiopatías congénitas
\end{tabular}

Tabla No. 3. Pacientes con corrección quirúrgica de cardiopatía.

\begin{tabular}{lcl}
\hline Cardiopatías operadas & Operados & Porcentaje \\
\hline *CIV & 8 & $22.8 \%$ \\
+PCA & 7 & $20 \%$ \\
-CIA & 7 & $20 \%$ \\
TETRALOGIA DE FALLOT & 7 & $20 \%$ \\
COARTACION DE LA AORTA & 4 & $11.5 \%$ \\
CIA + PCA & 2 & $5.7 \%$ \\
Total & 35 & $100 \%$ \\
\hline
\end{tabular}

*CIV: Comunicación interventricular, + PCA: persistencia del conducto arterioso, - CIA: comunicación interauricular Fuente: Investigación cardiopatías congénitas

\section{DISCUSIÓN}

Es imprescindible, como quedó patentado en la última Conferencia de Bethesda (32nd Bethesda Conference: Care of the Adult with Congenital Heart Disease), conocer la extensión del problema de las cardiopatías congénitas y para ello es básico, antes de realizar cualquier tipo de cálculo, identificar el número de niños con una cardiopatía congénita que nacen cada año en una población determinada. Debemos conocer la incidencia de las cardiopatías congénitas en nuestra población, y no sólo de forma global, sino desglosada por el tipo y severidad del defecto, lo que nos permitirá determinar los recursos necesarios y planificar su distribución de forma no sólo intuitiva. Si acudimos a la bibliografía, consta- 
taremos lo difícil que resulta obtener dichos datos. Los valores comunicados, procedentes de diversos estudios, son extraordinariamente variables. $^{(9)}$

Se encontró en este estudio que el sexo masculino fue el más afectado por cardiopatías congénitas representando el 53\% de los casos. Resultados similares se presentaron en investigación realizada en el Hospital Materno Infantil, Honduras en octubre del 2001 a mayo del 2003 donde el predominio de cardiopatías congénitas para el sexo masculino fue del $57.8 \%$. ${ }^{(10)}$ Difiere de datos estadísticos obtenidos en 2010 en Estados Unidos y Canadá donde las cardiopatías congénitas se presentaron con ligera predominancia en el sexo femenino. ${ }^{(11)}$

En este estudio la CIA constituyó el grupo más numeroso de cardiopatías congénitas, presente en $34 \%$ de los pacientes; estos datos concuerdan con los reportados a nivel mundial donde la CIA ha alcanzado alrededor del 50\% de ellas. ${ }^{(1,12)}$ Estudios realizados en el Hospital Nacional de niños de Costa Rica en 2006 reportaron que las cardiopatías más frecuentes fueron CIV (36\%) y CIA (20\%). ${ }^{(13)}$ Similar a los resultados de este estudio donde la segunda cardiopatía más frecuente fue CIV encontrada en $22.7 \%$ de los pacientes.

El 5\% (27) de malformaciones congénitas cardiovasculares presentaron relación con Síndrome de Down, coincidiendo con lo reportado en la literatura médica, incluso algunos autores plantean que estas cardiopatías son más frecuentes en pacientes con Síndrome de Down que en la población general. Además, la mitad de los pacientes presentaron cardiopatía congénita, con mayor frecuencia de canal auriculoventricular, comunicación interventricular y comunicación interauricular más comunicación interventricular, que se asociaron principalmente al síndrome de Down. ${ }^{(13-15)}$
En este estudio, se encontró que en los pacientes con Síndrome de Down la comunicación interauricular en conjunto con la persistencia del conducto arterioso estuvo presente en $40.7 \%$ y la comunicación interventricular en $26 \%$, dichos datos concuerdan con los resultados del estudio realizado en el Instituto Nacional de Pediatría de la ciudad de México en 2003, donde se estudiaron 275 niños con Síndrome de Down de los cuales las cardiopatías que se presentaron con mayor frecuencia fueron; la comunicación interauricular, comunicación interventricular y persistencia del ductus arterioso. ${ }^{(16,17)}$

En conclusión las frecuencias de las malformaciones congénitas encontradas en el estudio fueron similares a los reportados en otros países. El género masculino y los preescolares fueron los más afectados por cardiopatías congénitas, la comunicación interauricular correspondió a la cardiopatía más prevalente. Las anomalías cromosómicas tienen fuerte asociación con cardiopatías congénitas.

\section{RECOMENDACIONES}

Se recomienda implementar programas encargados de detección temprana de cardiopatías congénitas como oximetría del pulso como prueba de tamizaje en cardiopatías congénitas en neonatos apoyado con ecocardiografía para iniciar intervenciones oportunas evitando progresión al deterioro cardíaco en la población pediátrica.

Capacitación de personal médico para mejorar el diagnóstico prenatal y postnatal de cardiopatías congénitas favoreciendo a la derivación oportuna hacia los centros de referencia.

Se debe crear un registro nacional de cardiopatías congénitas para tener información de fácil acceso sobre el comportamiento de estas patologías en nuestro país. 


\section{BIBLIOGRAFÍA}

1. Heusser F. Problemas frecuentes en cardiología pediátrica. Rev chil pediatr. [Revista en internet] 2009 [Citado, 11 de Noviembre de 2015]; 1: 4-6. Disponible en: http://escuela. med.puc.cl/paginas/publicaciones/ma nualped/cardiocong.html

2. Working Group on Management of Congenital Heart Diseases in India. Consensus on timing of intervention for common congenital heart disease. Indian Pediatr. [Revista en internet] 2008[citado 10 de noviembre 2015]; 45(2):117-126. Disponible en: http:// www.ncbi.nlm.nih.gov/pubmed/18310790

3. Mendieta-Alcántara GG, Santiago-Alcántara E, Mendieta-Zerón H, Dorantes-Piña R, de Zárate-Alarcón GO, Otero-Ojeda GA. Incidencia de las cardiopatías congénitas y los factores asociados a la letalidad en niños nacidos en dos hospitales del Estado de México. Gaceta Med. De Mex. [Revista en internet] 2013 [Citado, 11 de Noviembre de 2015]; 149:617-623 Disponible en: http:// www.medigraphic.com/pdfs/gaceta/ gm-2013/gm136e.pdf.

4. Bonino Anna, Gómez Paula, Cetraro Laura, Etcheverry Gonzalo, Pérez Walter. Malformaciones congénitas: incidencia y presentación clínica. Arch. Pediatr. Urug. [revista en la Internet]. 2006 Oct [citado, 2015 Nov 11]; 77(3): 225-228. Disponible en: http:// www.scielo.edu.uy/scielo.php?script=sci arttext\&pid=S1688-12492006000300003 \&lng=es

5. Cajilema Herrera CK. Prevalencia de malformaciones congénitas en recién nacidos, en el área de neonatología del HRIA en el periodo comprendido de enero del 2008 a julio del 2009. Universidad Nacional de Loja. 2010 [Tesis] Disponible en http:// dspace.unl.edu.ec/jspui/bitstream/ 123456789/6605/1/Carmen\%20Karina\% 20Cajilema\%20Herrera.pdf
6. Penchaszadeh V. Nuevas tecnologías en reproducción y en ingeniería genética. En: Organización Panamericana de la Salud. Salud reproductiva de las Américas, Washington: OPS/OMS, 1992: 458-75.

7. De Sarasqueta, P. Mortalidad infantil por malformaciones congénitas y prematurez en la Argentina: análisis de los criterios de reducibilidad. Arch. Arg. de Ped [revista en la Internet]. 2006 Oct [citado, 2015 Nov 11]; 104(2): 153-158. Disponible en: http:// www.scielo.org.ar/scielo.php?script $=$ sci arttext\&pid=S0325-00752006000200012

8. Olórtegui A, Adrianzén M. Incidencia estimada de las cardiopatías congénitas en niños menores de 1 año en el Perú. Anales de la Fac. de Med. [revista en la Internet]. 2007 [citado, 2015 Nov 11];69(2):113-124. Disponible en: http://www.scielo.org.pe/ scielo.php?pid=S1025-55832007000 200003\&script=sci_arttext

9. Weeb GD, Williams RG.32nd Bethesda Conference: Care of the adult with congenital heart disease.J Am Coll Cardiol. [revista en la Internet]. 2001 [citado, 2015 Nov 11] 37(5): 1161-98. Disponible en: http://www.acha heart.org/Portals/0/pdf/BethesdaFull. pdf

10. Carvajal-Sierra P, Aronne-Gulllen E. Incidencia de cardiopatías congénitas en recién nacidos en el Hospital Materno Infantil diagnosticados entre octubre 2001 y mayo 2003. Rev. Med Hondur 2006; 85(5): 12-16.

11. Marelli A, Gilboa S, Devine O, Kucik J, Oster $M$, Riehle-Colarusso $T$, et al. Estimating the congenital heart disease population in the united states in 2010-what are the numbers?. Journal of the American College of Cardiology; [revista en Internet]. 2012 [citado, 2015 Nov 11];59 (13s1):E787-E787. Disponible en: http://content.onlinejacc. org/data/Journals/JAC/24309/07888.pdf 
2015 Nov 11] 30(1):31-38. Disponible en: http://www.scielosp.org/pdf/rpsp/v30n1/ v30n1a05.pdf

12. Schultz AH, Localio AR, Clark BJ, Ravishankar C, Videon N, Kimmel SE. Epidemiologic features of the presentation of critical congenital heart disease: implications for screening. Pediatrics; [Revista en Internet]. 2008 [citado, 2015 Nov 11];121(4) Disponible en: http://pediatrics.aappublications. org/content/121/4/751

13. Fonseca Hernández Mercedes, Pina Cobas Bertha, Acevedo Fonseca Raúl. Cardiopatías congénitas asociadas a cromosomopatías. Rev Cubana Pediatr [revista en la Internet]. 1997 Ago [citado 2015 Nov 17]; 69(2): 102-107. Disponible en: http://scielo.sld. $\mathrm{cu} /$ scielo.php? script $=$ sci_arttext $\&$ pid $=$ S0034-75311997000200005\&lng=es
14. Velasco Sánchez $R$, Santamaría Díaz H, Gómez Gómez M, Alva Espinosa C, Jiménez Arteaga S, Martínez Sánchez A. Cardiopatías congénitas y síndromes genéticos. Bol Med Hosp Infant Mex 1987;44(6):486-97.

15. Kuehl KS, Loffredo CA, Ferencz C. Failure to Diagnose Congenital Heart Disease in Infancy. Pediatrics. [Revista en Internet]. 1999 [citado, 2015 Nov 11];103(4) Disponible en: http://pediatrics.aappublications. org/content/103/4/743.full-text.pdf

16. De Rubens Figueroa, J., del Pozzo Magaña, B., Hach, J. L. P., Jiménez, C. C., \& Urbina, R. C. Malformaciones cardíacas en los niños con síndrome de Down. Revista española de cardiología. [Artículo en Internet]. 2003 [citado, 2015 Nov 11]; 56(9) Disponible en: http://www.revespcardiol.org/es/mal formaciones-cardiacas-los-ninos-con/arti culo/13051617/ 